\title{
Detección del estado fisiológico de los ojos en Conductores mediante técnicas de visión artificial
}

\author{
Computer vision techniques for detection of physiological status eyes drivers
}

\author{
Neisser Ale Ale ${ }^{1} \quad$ Junior Fabián ${ }^{1 *}$ \\ Recibido 08 de mayo de 2019, Aceptado 13 de junio de 2019 \\ Received: May 08, 2019 Accepted: June 13, 2019
}

\begin{abstract}
RESUMEN
En las últimas décadas, la cantidad de accidentes de tránsito debido a la fatiga o somnolencia del conductor ha provocado cuantiosas pérdidas humanas y materiales. A su vez, la venta en el parque automotor se ha masificado lo cual indica que posiblemente en los siguientes años si no se toma las medidas pertinentes para detectar la fatiga existirá un incremento en los accidentes automovilísticos. Este trabajo de investigación propone el desarrollo de un sistema de detección de fatiga en los conductores que permita alertar sobre sobre su estado mientras esté conduciendo mediante el uso de técnicas de visión artificial y machine learning. Las técnicas de estos dos campos de estudio se interceptan para generar modelos supervisados con un alto rendimiento al momento de clasificar el estado de fatiga en los conductores. En este estudio se ha trabajado con un dataset de imágenes frontales enfocándonos en la característica fisiológica de los ojos obteniendo resultados preliminares prometedores en la detección de fatiga en tiempo real.
\end{abstract}

Palabras clave: Detección de fatiga, visión artificial, machine learning, descriptor HOG, CEW dataset.

\section{ABSTRACT}

In recent decades, the number of traffic accidents due to fatigue or drowsiness of the driver has caused significant human and material losses. At the same time, the sale in the vehicle fleet has been massified, which indicates that possibly in the following years, if the pertinent measures are not taken to detect fatigue, there will be an increase in automobile accidents. Therefore, in this research study, the development of a fatigue detection system in drivers that allows alerting about their status while driving using artificial vision and machine learning techniques is proposed. The techniques of these two fields of study are intercepted to generate supervised models with high performance when classifying the state of fatigue in drivers. In this study, a dataset of frontal images focusing on the physiological characteristics of the eyes was used; obtaining promising preliminary results in the detection of fatigue in real-time.

Keywords: Fatigue detection, artificial vision, machine learning, HOG descriptor, CEW dataset.

\section{INTRODUCCIÓN}

En los últimos años la cantidad de accidentes automovilísticos se ha incrementado enormemente [14]. Uno de los factores es la imprudencia del conductor desencadenada por un estado de fatiga o somnolencia, que no es más que el hastío o cansancio de las personas ocasionado por el esfuerzo prolongado y que en algunas ocasiones produce alteraciones físicas [19]. En la mayoría de los casos esto se presenta en los conductores, puesto que, muchas veces recorren trayectos largos tanto por el día y noche, siendo este último el que mayor cansancio provoca por la ausencia de sueño. En tal

\footnotetext{
1 Universidad ESAN. Facultad de Ingeniería. Lima, Perú. E-mail: 13100884@ue.edu.pe; jfabian@esan.edu.pe

* Autor de correspondencia. E-mail: jfabian@esan.edu.pe
} 
sentido, en muchas investigaciones [13] indican que la mitad de los conductores evaluados tienen menos horas de sueño en el periodo de 24 horas, es decir, mayormente conducen el vehículo durante la noche sin dormir y eso hace que sientan mayor cansancio o fatiga en la madrugada [17].

En el Perú, el incremento de este tipo de accidentes ha generado alarmas de diversos organismos como por ejemplo el Ministerio de Transportes y Telecomunicaciones que actualmente pretende reducir la tasa de accidentes para el 2021, año del bicentenario de la independencia.

Las investigaciones en este campo enfocan cuatro categorías para la detección de la fatiga. La primera consiste en la señal fisiológica de los conductores, entre ellas electroencefalograma (EEG), electrocardiógrafo (ECG) y electrooculograma (EOG) $[18,16]$. Esta categoría da buenos resultados, pero obtener estas señales suele ser muy complicado y trabajoso. La segunda está basada en métodos de comportamiento de operación, por ejemplo, cuando el conductor está fatigado reduce su fuerza al sujetar el volante [15]. La tercera en métodos basados en el estado del vehículo, es decir, si está en el carril correcto o no. Por último, la cuarta basada en características fisiológicas [12] como parpadear y bostezar, pero sobre todo en el estado ocular. Muchos de estos métodos implican grandes costos, ya que, necesitan sensores, cámaras dentro y fuera del vehículo, así como un computador externo para un mejor procesamiento lo cual hace ineficiente la aplicación de estos métodos en un escenario real por resultar invasivos para el conductor.

Por lo anterior, este estudio tiene como objetivo el desarrollo de un sistema de detección de fatiga mediante el uso de técnicas de visión artificial y machine learning para la prevención de accidentes de tránsito basada en características fisiológicas de los conductores. Esto en base a que mayormente la medición de la fatiga [20] se hace con inspecciones visuales y la cual puede ser automatizada con la combinación de estas dos disciplinas.

La inteligencia artificial es parte fundamental de este sistema de detección de fatiga, ya que, este campo trata de construir entidades racionales, es decir, agentes que emprendan una acción ante una situación dada [10] mediante técnicas de machine learning. A su vez, otro módulo importante es el procesamiento de imágenes [11] que involucra mejorar la calidad de las imágenes usando técnicas de visión artificial para una mejor obtención de información e interpretación.

Para efectos de este estudio de investigación, hemos propuesto seguir una metodología que combine el uso de descriptores HOG para el procesamiento de las imágenes y la obtención de vectores característicos y el uso de clasificadores para el aprendizaje de los datos en base a si existe fatiga o no y así medir su rendimiento de acuerdo con la exactitud de la detección como métrica de evaluación.

Este artículo de investigación está estructurado de la siguiente manera: En la sección 2, se detallará la literatura que conlleva esta investigación tanto por parte de visión artificial como machine learning. Luego en la sección 3, se describirá la metodología y el impacto que tiene en la solución del problema planteado. A partir de la sección 4 y 5 se expondrá los resultados obtenidos mediante la experimentación con los datos y después se juzgará de acuerdo con los objetivos de esta investigación respectivamente. Por último, las conclusiones de este estudio se desprenderán en la sección 6.

\section{ANÁLISIS TEÓRICO}

Ya han pasado los años desde que Viola \& Jones [6] propusieron un clasificador de cara frontal para la detección de rostros, sin embargo, su precisión y rapidez han hecho que este clasificador basado en Adaboost sea muy utilizado para esta tarea. Su estructura se basa en clasificadores en cascada que recorren toda la imagen para determinar si existe por ejemplo cejas, ojos, nariz, boca, etc. tal como se muestra en la Figura 1.

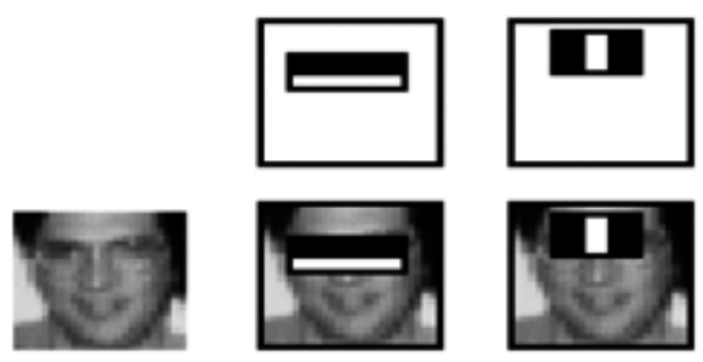

Figura 1. Clasificadores en Cascada de Viola \& Jones. 
Por otro lado, uno de los pasos para la clasificación y detección de imágenes es la extracción de características relevantes. Esta tarea consiste en transformar una imagen de entrada en un conjunto de características almacenados en un vector. Así pues, el principal objetivo es obtener la información más importante de una imagen y representarla en una dimensión reducida denominado vector característico [2]. Este vector servirá de entrada para cualquier clasificador haciendo que se maximice la tasa de detección de fatiga.

En este sentido tenemos a Histogram Oriented Gradients (HOG) que es un descriptor de características ampliamente usado para la detección de objetos a través de sus formas y texturas como el reconocimiento de logos en vehículos [4], así como para la detección del rostro o cuerpo humano. De acuerdo con el procedimiento para obtener el vector característico [1], el primer paso consiste en dividir la imagen de entrada en celdas, las cuales contienen cierto número de píxeles. Con estos se calcula los gradientes orientados y luego se construye el histograma de acuerdo con la magnitud de la posición del ángulo. Antes de obtener el vector final, se construye bloques, los cuales contienen celdas, para tener en cuenta los cambios en la iluminación y el contraste. Seguidamente, se une los histogramas de las celdas en cada bloque. La concatenación de los histogramas por bloque conforma el vector característico del descriptor HOG. La Figura 2 detalla este proceso.

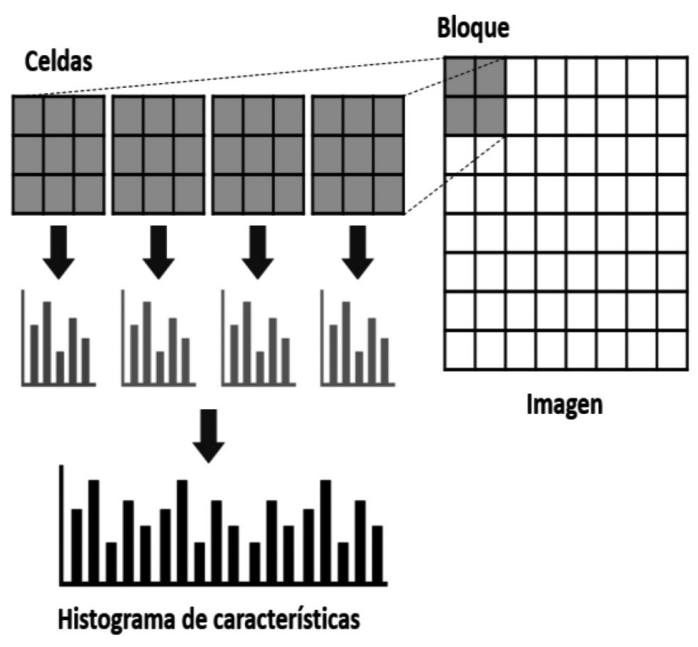

Figura 2. Esquema para obtener el descriptor de características HOG.
Por otro lado, existen diversos algoritmos para tareas de clasificación, regresión y agrupamiento que pertenecen al campo de machine learning. Enfocado en esta investigación se abordará aquellos específicos para la clasificación, puesto que, se pretende entrenar clasificadores con datos etiquetados. Alguno de ellos son Support Vector Machine (SVM), Random Forests y las Redes Neuronales. A continuación, se resume cada uno de ellos.

SVM o Máquina de Soporte Vectorial es una técnica de aprendizaje computacional formulada por Cortes \& Vapnik hace casi 50 años [21]. Sin embargo, no fue hasta las últimas décadas en que se hizo popular gracias al avance en potencia de hardware. La idea central de SVM es que, con un número suficientemente de dimensiones, siempre se puede encontrar un hiperplano que separa una clase particular de todas las demás. La Figura 3 detalla la estructura de un SVM para dos clases divididas por un hiperplano que trata de maximizar el margen a través de vectores de soporte más cercanos por cada clase y de una función en base a pesos.

Adicionalmente, Breiman [3] acuñó el término Random Forests o Bosques Aleatorios a un tipo de clasificador que agrupa un conjunto de árboles estructurados distribuidos de manera idéntica que arrojan un voto unitario para la clase más popular. Este algoritmo de aprendizaje supervisado usa el método bagging, el cual combina árboles de decisión con un set distinto de variables del mismo tamaño para obtener una mejor precisión y estabilidad y también diferentes muestras de los datos para cada árbol. Su uso es tanto para tareas de clasificación como regresión usando voto

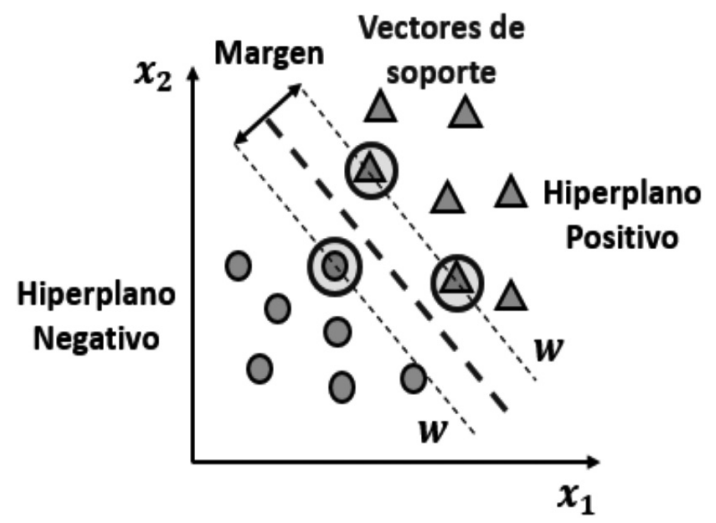

Figura 3. Arquitectura de $S V M$ para dos clases. 
mayoritario y ponderación respectivamente. La Figura 4 grafica el funcionamiento de esta técnica de machine learning.

Las redes neuronales también llamadas perceptrones multicapa se asemejan al comportamiento cuando las neuronas en nuestro cerebro intercambian información para comprender o percibir algo en nuestro entorno (también llamado sinapsis). Su arquitectura se basa en una capa de entrada, una capa oculta y capa de salida. La primera capa recibe los datos de entrada X que luego son multiplicados por pesos w de acuerdo con una función de activación $\mathrm{f}$, en la capa oculta, para emitir señales que activen a otras neuronas y obtener un resultado final en la última capa [9]. La arquitectura básica de una red neuronal se visualiza en la Figura 5.

Cabe resaltar que una red neuronal requiere más tiempo y potencia de CPU en comparación con otros modelos [8]. Sin embargo, son la base del éxito de

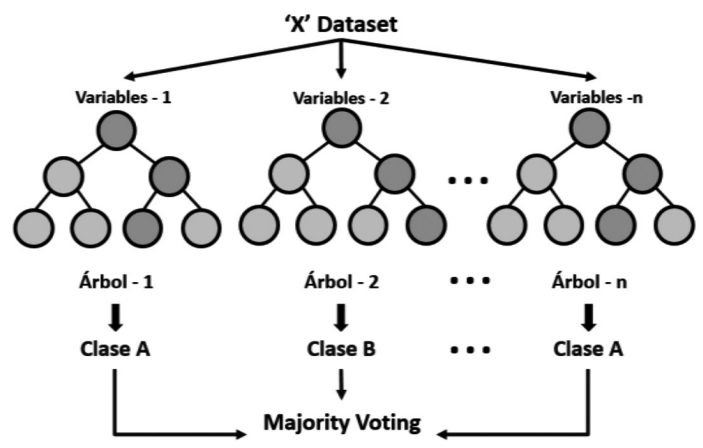

Figura 4. Arquitectura de Random Forests.

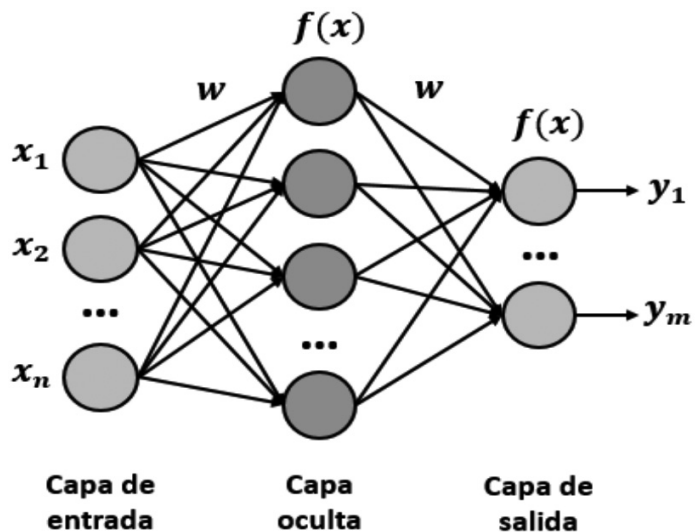

Figura 5. Arquitectura básica de las Redes Neuronales. la tecnología conocida como Deep Learning, ya que, este enfoque permite la clasificación, detección y segmentación de imágenes [7] de una manera muy precisa.

\section{METODOLOGÍA}

Python ofrece un conjunto de librerías esenciales para poder trabajar tanto para el procesamiento de imágenes y la clasificación. Así pues, se ha usado librerías como Numpy, Pandas, Scikit-learn y OpenCV orientado al trabajo con imágenes y entrenamiento de modelos supervisados con este lenguaje de programación muy usado en machine learning y data science.

El dataset para este trabajo de investigación se denomina Closed Eyes In The Wild (CEW) [5] el cual contiene imágenes de 2423 personas. De los cuales 1192 tienen los ojos cerrados y 1231 tienen los ojos abiertos. El dataset está dividido en dos tipos de imágenes por persona. La primera tiene como región de interés el rostro de los sujetos y la segunda está enfocada únicamente en los ojos como lo muestra la Figura 6. Esta última es la que ha sido utilizada para realizar los experimentos.

La metodología de esta investigación para el desarrollo de un sistema de detección se divide en dos fases para detectar si una persona está fatigada o no en base al estado fisiológico de los ojos.

\section{FASE DE APRENDIZAJE}

La primera se denomina Fase de Aprendizaje. Esta parte con la recolección de los datos del dataset de imágenes CEW correspondiente a individuos con los ojos abiertos y cerrados del lado izquierdo y derecho respectivamente. Luego para obtener el vector

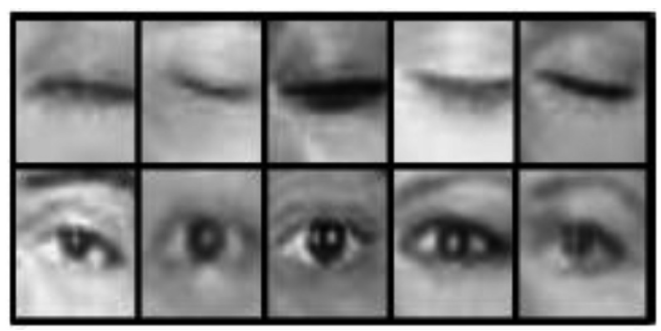

Figura 6. Imágenes 24 x 24 de CEW Dataset de ojos abiertos y cerrados. 
característico de las imágenes se aplica el descriptor HOG a la región de los ojos tanto derecho como izquierdo de manera independiente. Seguidamente, estos vectores son etiquetados y almacenados de acuerdo con el estado y posición del ojo, en otras palabras, como cerrado o abierto y derecho o izquierdo. A continuación, este dataset de características generado se divide en dos partes. La primera se denomina dataset de entrenamiento el cual contiene el $80 \%$ de los datos que sirven para entrenar un modelo de clasificación como Random Forests, SVM o Redes Neuronales. La segunda, es llamado dataset de prueba que contiene el $20 \%$ restante. Estos datos servirán para evaluar los modelos de clasificación mediante métricas, una de ellas la exactitud o accuracy para determinar el mejor modelo. Al final del proceso se tiene un clasificador que es capaz de detectar si un conductor tiene los ojos abiertos o cerrados. Por último, estos clasificadores son evaluados mediante la métrica de exactitud o accuracy para poder elegir el mejor modelo. La Figura 7 muestra nuestra propuesta del proceso de esta primera fase.

\section{FASE DE DETECCIÓN}

La segunda fase se denomina Fase de Detección. Para este proceso es necesario utilizar una cámara posicionada estratégicamente para capturar las imágenes frontales de los conductores cuando estén manejando. A estas imágenes se le aplica el clasificador en cascada de Viola \& Jones para detectar el rostro del conductor. Luego, se obtiene la región de interés, es decir, los dos ojos del rostro detectado. Con esta última región se obtiene los vectores característicos mediante el descriptor HOG usando los mismos parámetros de la Fase de Aprendizaje. Con estas nuevas características obtenidas de las imágenes enviamos a nuestros modelos (SVM, Random Forests o Redes Neuronales) que han sido previamente entrenado con la dataset de imágenes CEW, los cuales son capaces de detectar si el conductor está o no fatigado en base a la característica fisiológica de sus ojos mientras este está conduciendo el vehículo. La Figura 8 proyecta nuestra Fase de Detección.

\section{RESULTADOS}

Una vez concluida la fase de Aprendizaje con los parámetros respectivos para SVM (costo de 1000, kernel RBF y gamma de 0.001), Random Forests (100 árboles y 8 variables aleatorias por cada árbol) y la Red Neuronal (30 neuronas en la capa oculta y una función de activación relu), se ha evaluado los clasificadores según la métrica de exactitud o accuracy para determinar el mejor modelo usando

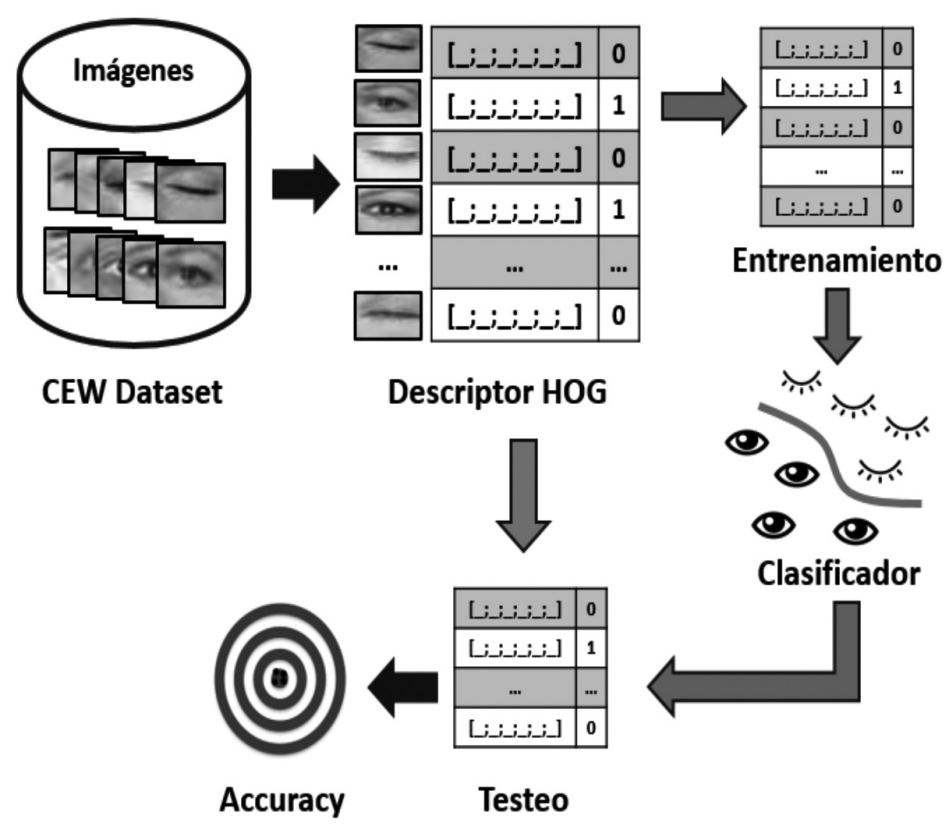

Figura 7. Fase de Aprendizaje del Sistema de detección de fatiga. 


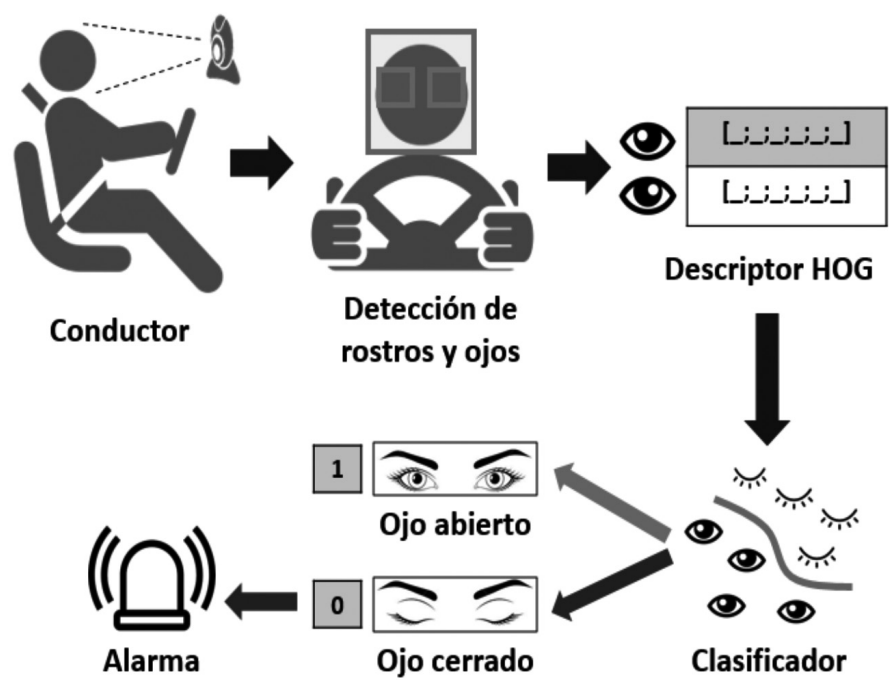

Figura 8. Fase de detección del sistema de detección de fatiga.

el descriptor de imágenes HOG. Estos resultados se muestran en la Tabla 1.

No obstante, existe un cuarto clasificador que se denomina Majority Voting. Este usa a los otros clasificadores para poder hacer un voto ensamblado según las predicciones de SVM, Random Forests y de la Red Neuronal para formar un propio clasificador y obtener una sola predicción. A continuación, en la Figura 9 se detalla esta estructura de clasificación. La Tabla 1 se divide en tres filas que corresponden al ojo derecho e izquierdo y al Mean Average Precision (mAP) que es el promedio de la métrica de exactitud o accuracy del clasificador en los dos ojos. Las columnas corresponden a los clasificadores usados para determinar el estado del ojo, es decir, si está abierto o cerrado. Los valores corresponden al factor de exactitud que conllevan los clasificadores.

Los resultados del clasificador generado por Majority Voting tuvieron un mejor rendimiento al momento de detectar el estado fisiológico del ojo tanto derecho como izquierdo en su estado de abierto o cerrado en comparación con los otros modelos con un mAP de $93,39 \%$. Esto dado que Majority Voting combina la potencia de SVM, Random Forests y la Redes Neuronales para mejorar la exactitud del modelo. Por otro lado, Random Forests trabaja mejor con los datos del ojo izquierdo con un accuracy de 93,60\% después del modelo Majority Voting con un accuracy de $94,22 \%$. El clasificador SVM tiene un bajo rendimiento en contraste con los demás modelos de clasificación, puesto que, obtiene los resultados más bajos con $91,75 \%$ y $92,98 \%$ para el ojo derecho e izquierdo respectivamente pero que aún resultan significativamente buenos en comparación con el estado del arte.

Al momento de la experimentación con un procesador Intel ${ }^{\circledR}$ Core ${ }^{\mathrm{TM}}$ i5 y con una memoria RAM de 4,00 GB el clasificador SVM obtiene mejores resultados en cuanto a rapidez con una velocidad

Tabla 1. Resultados de los clasificadores SVM, Random Forests, Redes Neuronales y Majority Voting usando el descriptor HOG para la detección del estado fisiológico de los ojos.

\begin{tabular}{|l|c|c|c|c|}
\hline \multicolumn{1}{|c|}{ Ojos } & SVM + HOG & RF + HOG & NN + HOG & Majority Voting \\
\hline Derecho & $91,75 \%$ & $92,57 \%$ & $92,16 \%$ & $92,57 \%$ \\
\hline Izquierdo & $92,98 \%$ & $93,60 \%$ & $92,57 \%$ & $94,22 \%$ \\
\hline mAP & $92,37 \%$ & $93,08 \%$ & $92,37 \%$ & $93,39 \%$ \\
\hline
\end{tabular}




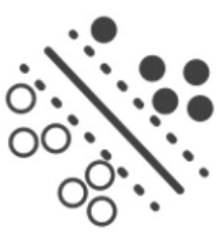

SVM

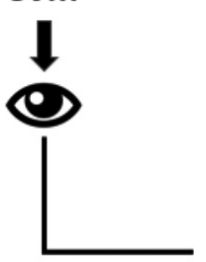

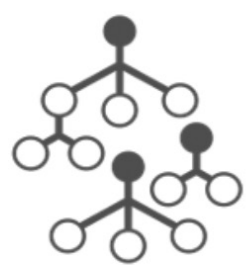

Random Forests

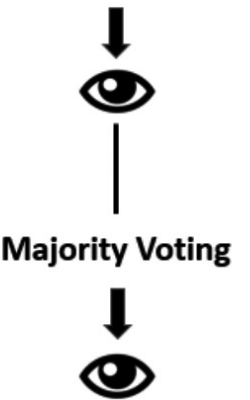

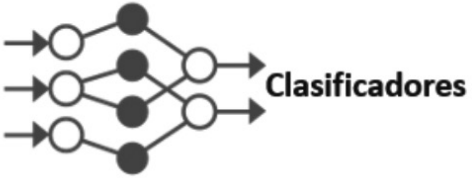

Neural Network

\section{Clasificación}

Múltiple

Clasificación

Final

Figura 9. Arquitectura de Majority Voting.

de 754 ms en comparación con los otros. Random Forests y las Redes Neuronales requieren de más gasto computacional y, por ende, demoran en la detección del estado del ojo en tiempo real con $791 \mathrm{~ms}$ y $878 \mathrm{~ms}$ respectivamente. Por el contrario, el clasificador Majority Voting es el que más tarda para realizar la detección con 908 ms, ya que, necesita de la combinación los anteriores clasificadores. La Tabla 2 muestra lo resultados indicando los tiempos por clasificador.

Tabla 2. Tiempo en que los clasificadores SVM, Random Forests, Redes Neuronales y Majority Voting tardan en la detección del estado fisiológico de los ojos en tiempo real.

\begin{tabular}{|l|c|}
\hline \multicolumn{1}{|c|}{ Clasificadores } & Tiempo en Detección \\
\hline SVM + HOG & $754 \mathrm{~ms}$ \\
\hline RF + HOG & $791 \mathrm{~ms}$ \\
\hline NN + HOG & $878 \mathrm{~ms}$ \\
\hline Majority Voting + HOG & $908 \mathrm{~ms}$ \\
\hline
\end{tabular}

Estas demoras pueden generar pérdidas de tiempo al momento en que el conductor se encuentra fatigado y conduciendo, ya que, esta situación es sensible y se requiere de rapidez para la predicción de la fatiga. Esto hará que aumente las probabilidades de evitar un accidente automovilístico de manera efectiva.
Por lo anterior, decidimos optar por SVM que obtiene una exactitud cercana a los demás clasificadores y es el que menos tiempo tarda en hacer la detección. Los resultados de detección usando SVM con el descriptor HOG de nuestro sistema propuesto se expone en la Figura 10.
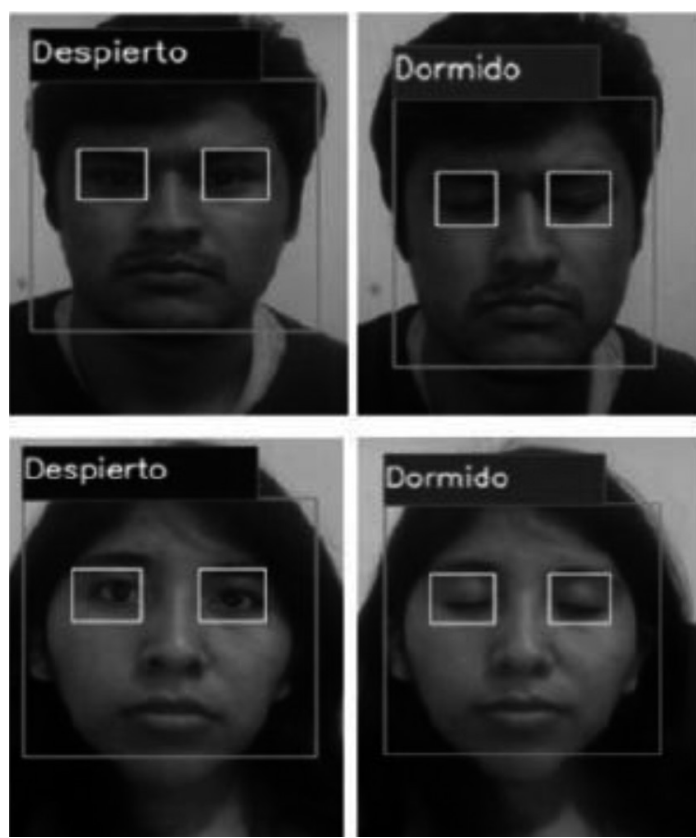

Figura 10. Detección del estado fisiológico de los ojos usando SVM y HOG en tiempo real. 
En la Figura 10 se ve claramente que SVM combinado con el descriptor de características HOG funciona correctamente y detecta si una persona esta con los ojos abiertos o cerrados etiquetándolos como si estuviesen despiertos o dormidos respectivamente en tiempo real.

\section{CONCLUSIONES}

Los campos de Visión Artificial y Machine Learning permiten el desarrollo de sistemas automatizados para la elaboración de tareas. En este estudio de investigación los abordó para tratar la problemática de seguridad vial. Más preciso aún para la detección de fatiga en los conductores. Demostrando que sí es posible aplicar las técnicas de visión artificial para esta tarea de predicción en una de las características fisiológicas de la fatiga como es el estado de los ojos.

Un Sistema de Detección de Fatiga puede reducir la cantidad de accidentes automovilísticos y por ende la reducción de pérdidas humanas y materiales. Ya que, una vez hecha la detección es factible añadir al Sistema de Detección de Fatiga un controlador o una alarma que despierte al conductor. Esta puede ser encender el radio, activar el sistema de ventilación o detener el vehículo.

Por otro lado, usar diversos clasificadores para la detección de fatiga en los conductores usando solamente la característica del estado de los ojos muestra resultados prometedores para el desarrollo de un sistema de detección de fatiga basada en estas características fisiológicas utilizando el descriptor HOG.

Sin embargo, las variaciones en la toma de las imágenes debido a la inclinación del conductor, la falta de iluminación, etc. hace que el Sistema encuentre más False Positives o False Negatives con mayor frecuencia. Esta limitación se puede solucionar con la calidad de resolución de la cámara o con características adicionales como las infrarrojas que ayudarían a controlar mejor el entorno y obtener más información.

Por último, es posible mejorar el entrenamiento con otras características fisiológicas como el bostezo o la inclinación de la cabeza del conductor. El procedimiento para el entrenamiento de los clasificadores en la Fase de Aprendizaje y para la
Fase de Detección sería la misma propuesta en esta investigación para la detección del estado del ojo.

\section{REFERENCIAS}

[1] N. Dalal and B. Triggs. "Histograms of Oriented Gradients for Human Detection". 2005 IEEE Computer Society Conference on Computer Vision and Pattern Recognition (CVPR'05). Vol. 1, pp. 886-893. 2005. DOI: 10.1109/CVPR.2005.177.

[2] G. Kumar and P. Kumar. "A Detailed Review of Feature Extraction in Image Processing Systems". 2014 Fourth International Conference on Advanced Computing \& Communication Technologies, pp. 5-12. 2014. DOI: 10.1109/ACCT.2014.74.

[3] L. Breiman. "Random Forest". Machine Learning. Vol. $45 \mathrm{~N}^{\mathrm{o}}$ 1, pp. 5-32. 2001. DOI: 10.1023/A:1010933404324.

[4] D. Llorca, R. Arroyo and M. Sotelo. "Vehicle logo recognition in traffic images using HOG features and SVM". 16th International IEEE Conference on Intelligent Transportation Systems (ITSC 2013), pp. 2229-2234. 2013. DOI: 10.1109/ ITSC.2013.6728559.

[5] F.Song, X.Tan, X.Liu and S.Chen. "Eyes Closeness Detection from Still Images with Multi-scale Histograms of Principal Oriented Gradients". Pattern Recognition. Vol. 47, pp. 2835-2838. 2014. DOI: 10.1016/j. patcog.2014.03.024.

[6] P. Viola and M. Jones. "Rapid object detection using a boosted cascade of simple features". Proceedings of the 2001 IEEE Computer Society Conference on Computer Vision and Pattern Recognition. CVPR 2001, Kauai, HI, USA, 2001.

[7] M. Mayank. "Ten Machine Learning Algorithms You Should Know to Become a Data Scientist". 2018 [online] https:// www.kdnuggets.com/2018/04/10-machinelearning-algorithms-data-scientist.html

[8] K. Soo. "Artificial Neural Networks (ANN) Introduction". Part 1. 2016 [online] https:// www.kdnuggets.com/2016/12/artificialneural-networks-intro-part-1.html

[9] S. Walczak. "Encyclopedia of Information Science and Technology". IGI Global. $\mathrm{N}^{\circ} 4$, pp. 120-131. 2018. 
[10] S. Russell and P. Norvig. "Artificial Intelligence. A modern approach". Pearson Education. $\mathrm{N}^{\circ}$ 3. pp. 1-34. Estados Unidos. 2010.

[11] G. Gómez y L. Sucar. "Visión Computacional". Departamento de Computación ITESM. $\mathrm{N}^{\mathrm{o}}$ 1. pp. 1-67. México. 2011.

[12] P. Sanghyuk,P. Fei, K. Sunghun and Chang. "Driver Drowsiness Detection System Based on Feature Representation Learning using Various Deep Networks". Computer Vision - ACCV 2016 Workshops. Vol. 10118, pp. 154-164. 2017. DOI: 10.1007/978-3-319-54526-4_12.

[13] G. Liendo, C. Castro y J. Rey de Castro. "Cansancio y somnolencia en los conductores de ómnibus interprovinciales, estudio comparativo entre formalidad e informalidad". Revista Peruana de Medicina Experimental y Salud Pública. Vol. 27, pp. 187-194. 2010. DOI: 10.1590/ S1726-46342010000200005.

[14] Instituto Nacional de Estadística e Informática. "Estadísticas de Seguridad Ciudadana". № 5 . Lima: Autor. 2017.

[15] L. Man and M. Hui-Ling. "A Method on Driver Fatigue Detection based on Multifeatures". International Journal of Signal Processing. Image Processing and Pattern Recognition.
Vol. $8 \mathrm{~N}^{\circ} 10$, pp. 107-114. 2015. DOI: 10.14257/ijsip.2015.8.10.12.

[16] W. Kong, L. Zhou, Y. Wang, J. Zhang, J. Liu and S. Gao. "A System of Driving Fatigue Detection Based on Machine Vision and Its Application on Smart Device". Journal of Sensor. Vol. 1, pp. 1-11. 2015. DOI: 10.1155/2015/548602.

[17] V. Saini and R. Saini. "Driver Drowsiness Detection System and Techniques: A Review". International Journal of Computer Science and Information Technologies. Vol. $5 \mathrm{~N}^{\mathrm{o}} 3$, pp. 4245-4249. 2014.

[18] F. Clement, A. Vashistha and M. Rane. "Driver Fatigue Detection System". 2015 International Conference on Information Processing (ICIP). pp. 229-234. 2015. DOI: 10.1109/INFOP.2015.7489384.

[19] Real Academia Española, Diccionario de la Lengua Española (23 $\left.{ }^{\mathrm{a}} \mathrm{Ed}.\right)$, 2014. [online] http://dle.rae.es/?id=HfqhQ.

[20] R. Jimenez. "Sistema de detección de nivel de cansancio en conductores mediante técnicas de visión por computador (Tesis de maestría, Universidad Nacional de Colombia, Bogotá, Colombia)". 2014.

[21] C. Cortes and V. Vapnik. "Support Vector Networks". Machine Learning. Vol. 20, pp. 273297. 1995. DOI: 10.1023/A:1022627411411. 